\title{
Improvement of Gender Balance Based on the Real- Time Visual Feedback System of the Pressure Center of Smart Wearable Devices: A Case Control Study
}

I-Lin Wang ( $\Delta$ ilin.wang@hotmail.com )

National Dong Hwa University https://orcid.org/0000-0002-6234-3768

Li-I Wang

National Dong Hwa University

Shi-Jie Xue

Jilin Sport University

Rui Hu

Jilin Sport University

Yu Su

Jilin Sport University

Rong-Jiun Jian

Lo-Hsu Medical Foundation Lotung Poh-Ai Hospital

Chun-Sheng HO

Lo-Hsu Medical Foundation Lotung Poh-Ai Hospital

Research

Keywords: gender difference, balance control, visual feedback, center of pressure

Posted Date: October 1st, 2020

DOI: https://doi.org/10.21203/rs.3.rs-78823/v1

License: (c) (i) This work is licensed under a Creative Commons Attribution 4.0 International License.

Read Full License 


\section{Abstract}

Background: The body maintains stability by integrating inputs from the central nervous system of vision, hearing, proprioception, and multiple senses. With the development of smart wearable devices, smart wearable devices can provide real-time center of pressure (COP) position-assisted balance control, which is beneficial to maintain physical balance.

Methods: Forty healthy college students (20 male-20 female) participated in this study, and the posture balance actions of left-leg stance non-visual feedback, left -leg stance visual feedback, right-leg stance non-visual feedback, and right-leg stance visual feedback are performed. Visual feedback provides smart insoles matching Podoon APP on a tablet computer with the COP position displayed by a dot as real-time visual feedback. A mixed-design two-way ANOVA was performed and included the study.

Results: The experimental results show that the displacement, velocity, radius, and area of the COP decreased significantly in the left-leg stance visual feedback/right-leg stance visual feedback, the test compared with the parameters in the eft-leg stance non-visual feedback/right-leg stance non-visual feedback $(P<0.05)$. Providing visual feedback through intelligent insoles can reduce the movement of the center of mass (COM) and maintain physical stability for healthy young people of different genders. In the one leg visual/non-visual in standing, the COP maximum anteroposterior displacement, COP anteroposterior velocity, COP radius, and COP area of women are significantly decreased than men $(\mathrm{P}<$ 0.05). Women have better real-time balance control ability than men with smart insoles.

Conclusion: The simple intelligent wearable assisted devices can immediately increase the control ability in static stance of men and women, and women have better real-time balance control ability than men.

\section{Background}

The body maintains its stability through its perception of space (i.e., balance ability) [1], and balance control is one of the important physical fitness in humans [2]. To maintain such a static balance, the body typically uses "hip strategy" or "ankle strategy" to project the center of mass (COM) in the base of support [3]. The COM sway and its spatial relationship with the base of support are the basis for balance control and performance [4]. Therefore, in the study of biomechanics, the change in the center of pressure (COP) in balance of systems is often considered as the main analysis parameter. The smaller the plantar COP displacement, the higher is the body's ability in maintaining a static balance [5]. In other words, a good control of the change in planar COP while maintaining a static balance will be beneficial to the performance of balance ability. Maintenance or improvement of balance is important for athletes, the elderly, those with ankle or knee injury history, and patients with sarcopenia.

Through the integration of vision, hearing, proprioception, and multiple sensory inputs from the central nervous system, organisms understand the relative movements of various parts of the body, the relationship between the body and the environment, and achieve the goal of stable standing by continuously adjusting muscle activity [6]. With regard to the distance between a space and an object, the 
prediction, direction, reaction time, time point, stability, and balance reflected by the brain are mainly provided by vision, and these factors significantly affect players' performances in competitions [7]. Therefore, the visual system is crucial for balance control. When moving, human beings often use the information provided by the visual system as a reference [8]. Real-time visual feedback (VF) can effectively reduce COP sway and accelerate posture control during standing posture balance [9]. Past studies used the Wii balance board real-time feedback function to improve postural control and balance in people with multiple disabilities [10]. VF can strengthen the body's control of COP displacement and reduce the movement of the body's COM, and finally achieve the rehabilitation effect of improving the body's balance [11]. Therefore, assisting proprioception through the balance board or VF system may improve body posture and stability during movement or balance control.

The body balance system is affected by many different factors, including age, height, BMI, and gender [12]. Gender differences exist in human postural stability. Past studies comparing boys and girls of the same age indicated that girls often demonstrated less postural sway [13], indicating gender differences in maintaining body balance. Furthermore, the study of gender differences in children's postural stability revealed that girls can integrate sensory input more effectively and rely more on somatosensory feedback than boys [14]. In addition, men are more likely to sway and cause physical instability than women because of their greater COP displacement during postural control [15]. Static and dynamic exercises show that women are excellent in maintaining static balance, affording posture stabilization and body balance maintenance [16]. Previous studies have shown that men have greater COP displacements after open-eyes/closed-eyes balance training, indicating that men are more unstable than women [17].

Therefore, the use of smart wearable devices to assist women or men with body posture control feedback will improve balance ability and women may perform better than men after VF.

In summary, COP change is an important index of balance control; it is an effective auxiliary method to provide real-time VF information. Smart wearable devices have been vigorously used in daily life. In the test of muscle fatigue in young healthy people, the vibration of insoles can compensate for the postural instability caused by fatigue and maintain the body balance [18]. Therefore, using real-time VF on the position of the COP provided by smart wearable devices effectively to maintain body balance can yield the benefits of technology-assisted homeostasis in maintaining body balance and help maintain body balance and physical rehabilitation. This study hypothesizes the following: 1) The balance posture ability of young people to maintain standing balance in VF is higher than non-visual feedback (NF). 2) When using smart insoles to provide VF, it is suitable for healthy people of different genders to improve physical stability. The aim of this study is to investigate the immediate effects of real-time VF information provided by smart insoles on the balance control ability of static balance movement. This study provides insights into the effects of real-time VF systems of the COP provided by smart wearable devices on the body's balance ability.

\section{Materials And Methods}

\section{Participants}


Forty healthy participants [20 males (age, 19.63 \pm 0.73 ; height, $1.67 . \pm 0.04$; weight, $58 \mathrm{~kg} \pm 6$; mean \pm SD) and 20 females (age, 20.74 \pm 0.98 ; height, 1.78. \pm 0.05 ; weight, $71 \mathrm{~kg} \pm 6$; mean \pm SD)] with no known balance pathology were included in the study. In addition, the inclusion criteria were normal visual acuity and contrast sensitivity. As required by the Helsinki Declaration, participants will be informed of the content, process, and precautions of the study group. They must understand and be willing to cooperate fully with the experimenter to sign the consent form. The study was approved by the Research Ethics Committee of Hualien Tzu Chi Hospital, Buddhist Tzu Chi Medical Foundation (IRB109-053-B).

\section{Procedures}

Prior to the experiment, the participants performed a 5-min warm-up run and a rested for $1 \mathrm{~min}$. The 40 participants completed the static balance ability test with eyes open under one-leg stance (OLS) with VF and NF: left-leg stance non-visual feedback (LLS-NF), left-leg stance visual feedback (LLS-VF), right-leg stance non-visual feedback (RLS-NF), and right-leg stance visual feedback (RLS-VF). During the static balance test, the participants were instructed to stand around the center above a force plate. The VF group used an iPad as an auxiliary device to achieve a stable body posture. The Podoon APP displayed the dynamic point of the COP, and the participants maintained the dynamic point in the central circle as much as possible. The data for each condition were collected for $30 \mathrm{~s}$ and three times. The static postural balance included the support leg standing upright, and the nonsupport leg was flexed at the knee of the support leg with the toes drooping naturally, the arms on the chest, and the palms on the shoulders. The iPad Pro used (iPad Pro, including the smart insoles APP) should be located at an eye-level height and 1.0 $\mathrm{m}$ apart from the participants. The NF group did not use the iPad VF.

\section{Equipment}

A force plate (BTS P6000, BTS Bioengineering, Italy) was used to collect the COP kinematics data: the $\mathrm{COP}$ maximum mediolateral displacement ( $\mathrm{COP}_{\mathrm{ML}}$ maximum displacement), $\mathrm{COP}$ maximum anteroposterior displacement ( $\mathrm{COP}_{\mathrm{AP}}$ maximum displacement), the $\mathrm{COP}$ mediolateral velocity $\left(\mathrm{COP}_{\mathrm{ML}}\right.$ velocity), $\mathrm{COP}$ anteroposterior velocity ( $\mathrm{COP}_{\mathrm{AP}}$ velocity), $\mathrm{COP}$ radius, and $\mathrm{COP}$ area. Force plate signals were collected at a sampling frequency of $600 \mathrm{~Hz}$. Each data point of the pretest and post-test was calculated from the average of three COPs.

\section{Statistical analysis}

Statistical analyses were performed using MATLAB (R2014a, The MathWorks, USA). A mixed-design twoway ANOVA was performed and included the following: two vision conditions (NF and VF) $\times$ gender (woman and man) were used to compare the $\mathrm{COP}_{\mathrm{ML}} / \mathrm{COP}_{\mathrm{AP}}$ maximum displacement, $\mathrm{COP}_{\mathrm{ML}} / \mathrm{COP}_{\mathrm{AP}}$ velocity COP radius, and COP area. The statistical significance level was set at $a=.05$. Intraclass correlation coefficients (ICC) were calculated for the COP parameters in the NF and VF, as poor (ICC < $0.40)$, moderate $(0.40 \leq \mathrm{ICC}<0.60)$, good $(0.60 \leq \mathrm{ICC}<0.80)$, and excellent $(\mathrm{ICC} \geq 0.80)$ [19]. The ICC ranged from 0.64 to 0.96 . 


\section{Results}

\section{Gender differences and vision conditions in $\mathrm{COP}_{\mathrm{ML}}$ and $\mathrm{COP}_{\mathrm{AL}}$ maximum displacement parameter analysis}

The training results of the gender differences and vision conditions in $\mathrm{COP}_{\mathrm{ML}}$ and $\mathrm{COP}_{\mathrm{AL}}$ maximum displacement parameter are shown in Fig. 1.The interaction (vision conditions $\times$ gender) of the COPML maximum displacement or $\mathrm{COP}_{\mathrm{AP}}$ maximum displacement in the RLS did not differ significantly $(\mathrm{p}=$ 0.157 and $p=0.222$ ); The $\mathrm{COP}_{M L}$ maximum displacement differed significantly in the vision conditions ( $p$ $<0.001)$; the analysis of vision conditions indicated a significant decrease in RLS-VF-Man/Woman compared with RLS-NF-Man/Woman $(\nabla 9.56 \%$ and $\nabla 15.60 \%)$. The main effect of the $\mathrm{COP}_{\mathrm{AP}}$ maximum displacement differed significantly by gender $(p=0.013)$; the analysis of gender indicated a significant decrease in RLS-VF/NF-Woman compared with RLS-VF/NF-Man ( $\nabla 16.17 \%$ and $\nabla 22.42 \%)$. The vision conditions differed significantly $(\mathrm{p}<0.001)$, the analysis of vision conditions indicated a significant decrease in RLS-VF-Man/Woman compared with RLS-NF-Man/Woman ( $\nabla 22.24 \%$ and $\nabla 15.96 \%)$. The interaction (vision conditions $\times$ gender) of the $\mathrm{COP}_{\mathrm{ML}}$ maximum displacement or $\mathrm{COP}_{\mathrm{AP}}$ maximum displacement in the left-leg stance (LLS) did not differ significantly $(p=0.315$ and $p=0.703)$. The main effect of the $\mathrm{COP}_{\mathrm{ML}}$ maximum displacement was a significant difference in the vision conditions $(p$ $<0.001)$; the analysis of vision conditions indicated a significant decrease in LLS-VF-Man/Woman compared with LLS-NF-Man/Woman $(\nabla 14.28 \%$ and $\nabla 9.93 \%)$. The main effect of the COP $_{\text {AP }}$ maximum displacement differed significantly by gender $(p=0.007)$; the analysis of gender showed a significant decrease in LLS-VF/NF-Woman compared with LLS-VF/NF-Man ( $\nabla 13.67 \%$ and $\nabla 13.40 \%$ ); the vision conditions showed a significant difference $(p<0.001)$; the analysis of vision conditions showed a significant decrease in LLS-VF-Man/Woman compared with LLS-NF-Man/Woman $(\nabla 21.80 \%$ and $\nabla 20.78 \%)$. Therefore, the $\mathrm{COP}_{\mathrm{ML}}$ maximum displacement or $\mathrm{COP}_{\mathrm{AP}}$ maximum displacement of men/women decreased significantly in the VF group compared with the NF group, whereas the $\mathrm{COP}_{\mathrm{AP}}$ maximum displacement of women was significantly lower than that of men in each balance condition.

\section{Gender differences and vision conditions in $\mathrm{COP}_{\mathrm{ML}}$ and $\mathrm{COP}_{\mathrm{AL}}$ velocity parameter analysis}

The training results of the gender differences and vision conditions in $\mathrm{COP}_{\mathrm{ML}}$ and $\mathrm{COP}_{\mathrm{AL}}$ velocity parameter are shown in Fig. 2. The interaction (vision conditions $\times$ gender) of the $\mathrm{COP}_{\mathrm{ML}}$ velocity or $\mathrm{COP}_{\mathrm{AP}}$ velocity in the RLS did not differ significantly $(p=0.895$ and $p=0.534)$. The main effect of the $\operatorname{COP}_{M L}$ velocity was a significant difference in the vision conditions $(p=0.001)$; the analysis of vision conditions showed a significant decrease in RLS-VF-Man/Woman compared with RLS-NF-Man/Woman $(\nabla 13.43 \%$ and $\nabla 12.43 \%)$. The main effect of the $\mathrm{COP}_{\mathrm{AP}}$ velocity was a significant difference in gender ( $\mathrm{p}$ $<0.001)$; the analysis of gender showed a significant decrease in RLS-VF/NF-Woman compared with RLS-VF/NF-Man $(\nabla 15.20 \%$ and $\nabla 22.44 \%)$. The vision conditions differed significantly $(p=0.001)$; the analysis of vision conditions showed a significant decrease in RLS-VF-Man/Woman compared with RLSNF-Man/Woman $(\nabla 5.42 \%$ and $\nabla 8.69 \%)$. The interaction (vision conditions $\times$ gender) of the $\mathrm{COP}_{\mathrm{ML}}$ 
velocity or $\mathrm{COP}_{A P}$ velocity in the LLS did not differ significantly $(p=0.770$ and $p=0.139)$. The main effect of the $\mathrm{COP}_{M L}$ velocity was a significant difference in vision conditions $(p=0.001)$, analysis of vision conditions was a significant decrease in LLS-VF-Man/Woman compared with LLS-NF-Man/Woman $(\nabla 7.18 \%$ and $\nabla 8.39 \%)$. The main effect of the $\mathrm{COP}_{\mathrm{AP}}$ velocity was a significant difference in gender ( $\mathrm{p}=$ $0.020)$; the analysis of gender showed a significant decrease in LLS-VF/NF-Woman compared with LLSVF/NF-Man $(\nabla 10.06 \%$ and $\nabla 6.49 \%)$. The vision conditions differed significantly $(p<0.001)$; the analysis of vision conditions showed a significant decrease in LLS-VF-Man/Woman compared with LLS-NFMan/Woman $(\nabla 2.62 \%$ and $\nabla 6.82 \%)$. Therefore, the $\mathrm{COP}_{\mathrm{ML}}$ velocity or $\mathrm{COP}_{\mathrm{AP}}$ velocity of men/women decreased significantly decreased in the VF group compared with the NF group, and the $\mathrm{COP}_{\mathrm{AP}}$ velocity of women was significantly lower than that of men in each balance condition. This shows that women have better balance control ability than men.

\section{Gender differences and vision conditions in COP radius and COP area parameter analysis}

The training results of the gender differences and vision conditions in COP radius and COP area parameter are shown in Fig. 3. The interaction (vision conditions $\times$ gender) of the COP radius or COP area in the RLS did not differ significantly $(p=0.439$ and $p=0.241)$. The main effect of the COP radius was a significant difference in gender $(p=0.021)$; the analysis of gender showed a significant decrease in RLSVF/NF-Woman compared with RLS-VF/NF-Man ( $\nabla 14.10 \%$ and $\nabla 17.78 \%)$. The vision conditions differed significantly $(p=0.001)$; the analysis of vision conditions showed a significant decrease in RLS-VFMan/Woman compared with RLS-NF-Man/Woman $(\nabla 13.33 \%$ and $\nabla 21.80 \%)$. The main effect of the COP area was a significant difference in gender $(p=0.001)$; the analysis of gender showed a significant decrease in RLS-VF/NF-Woman compared with RLS-VF/NF-Man ( $\nabla 30.16 \%$ and $\nabla 33.44 \%$ ). The vision conditions differed significantly $(\mathrm{p}=0.001)$; the analysis of vision conditions showed a significant decrease in RLS-VF-Man/Woman compared with RLS-NF-Man/Woman ( $\nabla 21.16 \%$ and $\nabla 16.84 \%)$. The interaction (vision conditions $\times$ gender) of the COP radius or COP area in the LLS did not differ significantly $(p=0.945$ and $p=0.745)$. The main effect of the COP radius was a significant difference in gender $(p=0.003)$; the analysis of gender showed a significant decrease in LLS-VF/NF-Woman compared with LLS-VF/NF-Man $(\nabla 11.49 \%$ and $\nabla 9.37 \%)$. The vision conditions differed significantly $(p<0.001)$; the analysis of vision conditions showed a significant decrease in LLS-VF-Man/Woman compared with LLSNF-Man/Woman ( $\nabla 9.37 \%$ and $\nabla 11.49 \%)$. The main effect of the COP area was a significant difference in gender $(p=0.020)$; the analysis of gender showed a significant decrease in LLS-VF/NF-Woman compared with LLS-VF/NF-Man ( $\nabla 18.22 \%$ and $\nabla 18.32 \%)$. The vision conditions differed significantly $(p=0.002)$; the analysis of vision conditions showed a significant decrease in LLS-VF-Man/Woman compared with LLS-NF-Man/Woman ( $\nabla 11.93 \%$ and $\nabla 14.21 \%)$. Therefore, the COP radius or COP area of men/women decreased significantly in the VF group compared with the NF group, and the COP radius and COP area of women decreased significantly decreased compared with that of men in each balance condition.

\section{Discussion}


The purpose of this study is to investigate the auxiliary effect of VF provided by smart insoles on realtime static balance control in healthy young people of different genders. The results showed that the displacement, velocity, radius, and area of COP decreased significantly, whereas those of men or women in OLS-VF, and the $\mathrm{COP}_{\mathrm{AP}}$ displacement, $\mathrm{COP}_{\mathrm{AP}}$ velocity, COP radius, and COP area of women decreased significantly decreased. Furthermore, the COP parameter decreased as the body stability strengthened [5]. The VF real-time static balance ability provided by smart insoles was higher than that of NF, and the realtime balance control ability of women was higher than that of men.

After VF was provided, the $\mathrm{COP}_{\mathrm{AP}}$ maximum displacement, $\mathrm{COP}_{\mathrm{AP}}$ variance, $\mathrm{COP}_{\mathrm{ML}}$ maximum displacement, and $\mathrm{COP}_{\mathrm{ML}}$ variance reduced, indicating the effectiveness of $\mathrm{VF}$ as a posture control strategy [9]. The direction of the COP track shows different control strategies for postural sway during standing. The postural sway in the mediolateral $(M L)$ direction is related to hip joint stability, whereas the postural sway in the anteroposterior (AP) direction is related to ankle joint stability [20]. In the AP direction, body sway and posture changes are closely related to the ankle neuromuscular function. The "ankle strategy" can increase the stability of the ankle joint and reduce the COP displacement to improve the balance ability [21]. In this study, the decrease in COP displacement in the AP direction may be due to the effect of VF on the stability of the ankle joint. Static balance can be maintained through an active torque regulation/control of sensory motor integration for muscle contraction during ankle position modulation [7]. Therefore, the intervention of VF enables the COP to be displayed on the screen, and the body actively controls the COP according to the change in the COP to maintain physical stability. The central nervous system is fully involved in regulating the movement of the ankle muscles to maintain the stability of the COM. In this study, the $\mathrm{COP}_{\mathrm{ML}}$ maximum displacement decreased after VF intervention. Previous studies revealed that the COP displacement improved in both the and AP directions during the exercise of stable static balance [22]. When controlling the ML displacement, the "hip strategy" accompanied by VF control balance results in autonomous adduction and abduction [23]. Moreover, the displacement in the $\mathrm{ML}$ direction can improve balance ability through this autonomous adduction and abduction change. Therefore, static balance standing after VF may cause the joint activity of "ankle strategy" and "hip strategy" to maintain stability, thereby reducing the degree of body sway and displacement variation after VF.

The COP mean velocity, $\mathrm{COP}_{\mathrm{AP}}$ velocity, and $\mathrm{COP}_{\mathrm{ML}}$ velocity decreased after VF intervention. Previous studies regarding the balance of soccer players revealed that the decrease in COP velocity was due to the more effective prediction of body position changes by the visual system of soccer players [7]. The difference in parameters before and after the experiment in this study may be caused by the VF information obtained after VF intervention to predict body position changes. The decrease in the $\mathrm{COP}_{\mathrm{AP}}$ velocity may be due to the body's assessment of body status through VF to achieve better stability [7]. When standing, the body feeds back position and sensory information to the central nervous system to control the muscle activity of the ankle joint mechanism and facilitate the body in maintaining balance. In the OLS, the $\mathrm{COP}_{\mathrm{AP}}$ velocity controlled by the "ankle strategy" and the $\mathrm{COP}_{\mathrm{ML}}$ velocity controlled by the "hip strategy" decreased, thereby increasing the stability of posture control [24].The maintenance of static 
balance in the human body is typically completed by the activities of the trunk and lower limb muscles, such as the "hip strategy" or "ankle strategy" [3]. Therefore, the decrease in the $\mathrm{COP}_{\mathrm{AP}}$ velocity and COP $\mathrm{ML}$ velocity may be the result of the mediation of joint motion between the ankle and hip after VF, which can enhance the stability of the lower extremities.

The COP area and COP radius under VF decreased, indicating that the stability of the participants may increase after VF intervention. Previous studies revealed that using VF and virtual reality balance tests can reduce the COP radius and area [25]. Therefore, the enhancement of VF will strengthen the participants' ability in controlling balance. Balance control is a dynamic adaptation process of active (autonomous control) and passive (accidental, external disturbance) balance control actions [26]. When visual information increases, the body's autonomous control ability is strengthened [27]. Moreover, the enhancement in autonomous control ability reduces the displacement area and COP radius to strengthen the physical stability [8]. The decrease in COP area and COP radius in this study may be due to the increase in the autonomous control ability of VF for enhancing physical stability. In addition, previous studies revealed that healthy adults under VF maintained body balance more effectively [28]. Therefore, visual information strengthens the sensory system of young people and increases the ability of autonomous control under the regulation of the central nervous system to enhance physical stability.

Previous studies revealed that the COP displacement of men was greater than that of women [17], which is consistent with the results of this study, i.e., the $\mathrm{COP}_{\mathrm{AP}}$ displacement of men is greater than that of women. The sway lengths of men and women were longer in the AP direction than in the ML direction, and the displacement range of men was larger; women indicated a stronger ability to perceive surrounding information and effectively integrated perceptual information [29]. In addition, the difference in height and weight between men and women caused the displacement range of men to be greater than that of women [17]. Therefore, in this study, women used the surrounding sensory system to integrate movement and promote greater stability. The $\mathrm{COP}_{\mathrm{AP}}$ velocity of men in this study was greater than that of women, which may be due to the higher upper body weight of men, resulting in a higher relative vertical position of the COM [17]. This will affect the projection of the COM and reduce the physical stability. In the absence of sensory aids, the posture control ability of men was worse [30]. Therefore, the lower COM of women with the participation of intelligent auxiliary equipment is beneficial for controlling the stability of the body. The COP radius and COP area of both women and men decreased after VF intervention, whereby those of women decreased more than those of men. Compared with men, women have less motor function, are more sensitive to surrounding information, and exhibit higher autonomous ability in balance control [30]. Therefore, smart insoles can increase VF and maintain physical stability. During balance control, women use the surrounding sensory information to increase autonomous control under the regulation of the central nervous system, thereby improving the stability of static standing. The limitations of this study were that the EMG and kinematic images of participants were not collected; moreover, it was difficult to detect the balance regulation of joint muscle vision conditions and joint angle. Therefore, the application of smart wearables in several weeks of balance training to assist the 
body to improve balance should be applied to individuals with unstable postures in future studies to investigate the benefits of VF on balance improvement.

\section{Study limitations}

The limitation of this study is that there is no comparison of age balance performance differences. Maturational processes also affecting the development of balance performance in youth differ in onset and velocity inter individually, consequently complicating comparisons based on chronological age only. future studies on children and adolescents of different ages should be compared in terms of their physiological age balance to explore their athletic performance.

\section{Conclusions}

Intelligent auxiliary equipment increases the participants' visual information, and the increase in VF may promote the integration of the central nervous system to increase the body's real-time postural stability. When a smart auxiliary equipment is applied to VF to increase visual information, it can increase the autonomous control ability of young people. It is beneficial for the central nervous system to control the balance mechanism to perform autonomous adjustments to maintain physical stability, and it would be better for women to control the sensory information around them autonomously. In this study, a simple smart auxiliary equipment was used to provide VF information of the COM to maintain the real-time balance ability of healthy people of different genders for static standing. Women demonstrated higher real-time balance ability in VF than men. Moreover, the use of smart insoles to provide VF yielded intuitive, simple, and real-time feedback with strong operability. In the future, this technology can be further extended to various rehabilitation purposes or improve the balance of the human body.

\section{Declarations}

\section{Ethical approval}

The study was approved by the Research Ethics Committee of Hualien Tzu Chi Hospital, Buddhist Tzu Chi Medical Foundation (IRB109-053-B).

\section{Consent for publication}

Not applicable.

\section{Availability of data and materials}

The datasets during and/or analyzed during this study are available from the corresponding author on reasonable request.

\section{Competing interests}


There were no conflicts of interest with other authors or institutions for this study.

\section{Funding}

This research received grant from the Lo-Hsu Medical Foundation, Inc., Lotung Poh-Ai Hospital (Number: E157).

\section{Authors' contributions}

SJX, YL, JQL, and RH prepared the manuscript. ILW and YMC contributed to the experimental design and balance training exercise program. ILW, SJX, YL, and RH analyzed the data to describe the effects of body movement on VF training. SJX and YL analyzed the effects of training under the COP parameters between the pretest and post-test. ILW reviewed and edited the manuscript. All authors have read and approved the final manuscript.

\section{Acknowledgments}

The authors thank Shi-Jie Xue and Yang Liu for conducting the biochemical examinations. This study was supported by the Research Ethics Committee of Hualien Tzu Chi Hospital, Buddhist Tzu Chi Medical Foundation.

\section{References}

1. Hrysomallis C. Balance ability and athletic performance. Sports med. 2011;41(3):221-32.

2. Goodway JD, Ozmun JC, Gallahue DL. Understanding motor development: Infants, children, adolescents, adults: Jones \& Bartlett Learning; 2019.

3. Horak F, Kuo A. Postural adaptation for altered environments, tasks, and intentions. Biomechanics and neural control of posture and movement: Springer; 2000. p. 267-81.

4. Lizama LEC, Pijnappels M, Reeves NP, Verschueren SM, van Dieën JH. Centre of pressure or centre of mass feedback in mediolateral balance assessment. Journal of biomechanics. 2015;48(3):539-43.

5. Stanković M, Radenković 0 . The status of balanve in preschool children involved in dance program. Research in Kinesiology. 2012;40(1).

6. Brauer SG, Woollacott M, Shumway-Cook A. The interacting effects of cognitive demand and recovery of postural stability in balance-impaired elderly persons. The Journals of Gerontology Series A: Biological Sciences and Medical Sciences. 2001;56(8):M489-M96.

7. Thompson LA, Badache M, Cale S, Behera L, Zhang N. Balance Performance as Observed by Centerof-Pressure Parameter Characteristics in Male Soccer Athletes and Non-Athletes. Sports (Basel, Switzerland). 2017;5(4).

8. Stins J, Michielsen M, Roerdink M, Beek PJJG, Posture. Sway regularity reflects attentional involvement in postural control: Effects of expertise, vision and cognition. 2009;30(1):106-9. 
9. Kręcisz K, Kuczyński M. Attentional demands associated with augmented visual feedback during quiet standing. PeerJ. 2018;6:e5101.

10. Shih $\mathrm{C}-\mathrm{H}$, Shih $\mathrm{C}-\mathrm{T}$, Chiang M-S. A new standing posture detector to enable people with multiple disabilities to control environmental stimulation by changing their standing posture through a commercial Wii Balance Board. Research in Developmental Disabilities. 2010;31(1):281-6.

11. Rougier P, Farenc I, Berger L. Modifying the gain of the visual feedback affects undisturbed upright stance control. Clin Biomech. 2004;19(8):858-67.

12. Olchowik G, Tomaszewski M, Olejarz P, Warchoł J, Różańska-Boczula M, Maciejewski R. The human balance system and gender. Acta of bioengineering and biomechanics. 2015;17(1).

13. Lee AJ, Lin W-H. The influence of gender and somatotype on single-leg upright standing postural stability in children. Journal of applied biomechanics. 2007;23(3):173-9.

14. Smith A, Ulmer F, Wong D. Gender differences in postural stability among children. Journal of human kinetics. 2012;33(1):25-32.

15. Steindl R, Kunz K, Schrott-Fischer A, Scholtz A. Effect of age and sex on maturation of sensory systems and balance control. Developmental medicine and child neurology. 2006;48(6):477-82.

16. Schedler S, Kiss R, Muehlbauer T. Age and sex differences in human balance performance from 6-18 years of age: A systematic review and meta-analysis. PLoS one. 2019;14(4):e0214434.

17. Bryant EC, Trew ME, Bruce AM, Kuisma RM, Smith AW. Gender differences in balance performance at the time of retirement. Clinical biomechanics (Bristol, Avon). 2005;20(3):330-5.

18. Moon J, Pathak P, Kim S, Roh S-G, Roh C, Shim Y, et al. Shoes with active insoles mitigate declines in balance after fatigue. Sci Rep. 2020;10(1):1951-.

19. Mancini M, Salarian A, Carlson-Kuhta P, Zampieri C, King L, Chiari L, et al. ISway: a sensitive, valid and reliable measure of postural control. 2012;9(1):59.

20. Mochizuki G, Semmler JG, Ivanova TD, Garland SJ. Low-frequency common modulation of soleus motor unit discharge is enhanced during postural control in humans. Experimental brain research. 2006;175(4):584-95.

21. Park KH, Lim JY, Kim TH. The effects of ankle strategy exercises on unstable surfaces on dynamic balance and changes in the COP. Journal of physical therapy science. 2016;28(2):456-9.

22. Kaji A, Sasagawa S, Kubo T, Kanehisa H. Transient effect of core stability exercises on postural sway during quiet standing. Journal of strength and conditioning research. 2010;24(2):382-8.

23. Winter DA, Prince F, Frank JS, Powell C, Zabjek KF. Unified theory regarding A/P and M/L balance in quiet stance. Journal of neurophysiology. 1996;75(6):2334-43.

24. Koyama K, Yamauchi J. Altered postural sway following fatiguing foot muscle exercises. PLoS One. 2017;12(12):e0189184.

25. Ravi DK, Kumar N, Singhi P. Effectiveness of virtual reality rehabilitation for children and adolescents with cerebral palsy: an updated evidence-based systematic review. Physiotherapy. 2017;103(3):24558. 
26. Gefen R, Dunsky A, Hutzler YJPt. Balance training using an iPhone application in people with familial dysautonomia: three case reports. 2015;95(3):380-8.

27. DG S, MI A, K M, AH V, H O, MR P, et al. Positive effect of balance training with visual feedback on standing balance abilities in people with incomplete spinal cord injury. Spinal cord. 2010;48(12):88693.

28. S B, PR R. Relation between postural control assessment with eyes open and centre of pressure visual feedback effects in healthy individuals. Experimental brain research. 2009;195(1):145-52.

29. Sullivan EV, Rose J, Rohlfing T, Pfefferbaum A. Postural sway reduction in aging men and women: relation to brain structure, cognitive status, and stabilizing factors. Neurobiology of aging. 2009;30(5):793-807.

30. Steindl R, Kunz K, Schrott-Fischer A, Scholtz AW. Effect of age and sex on maturation of sensory systems and balance control. Dev Med Child Neurol. 2006;48(6):477-82.

\section{Figures}




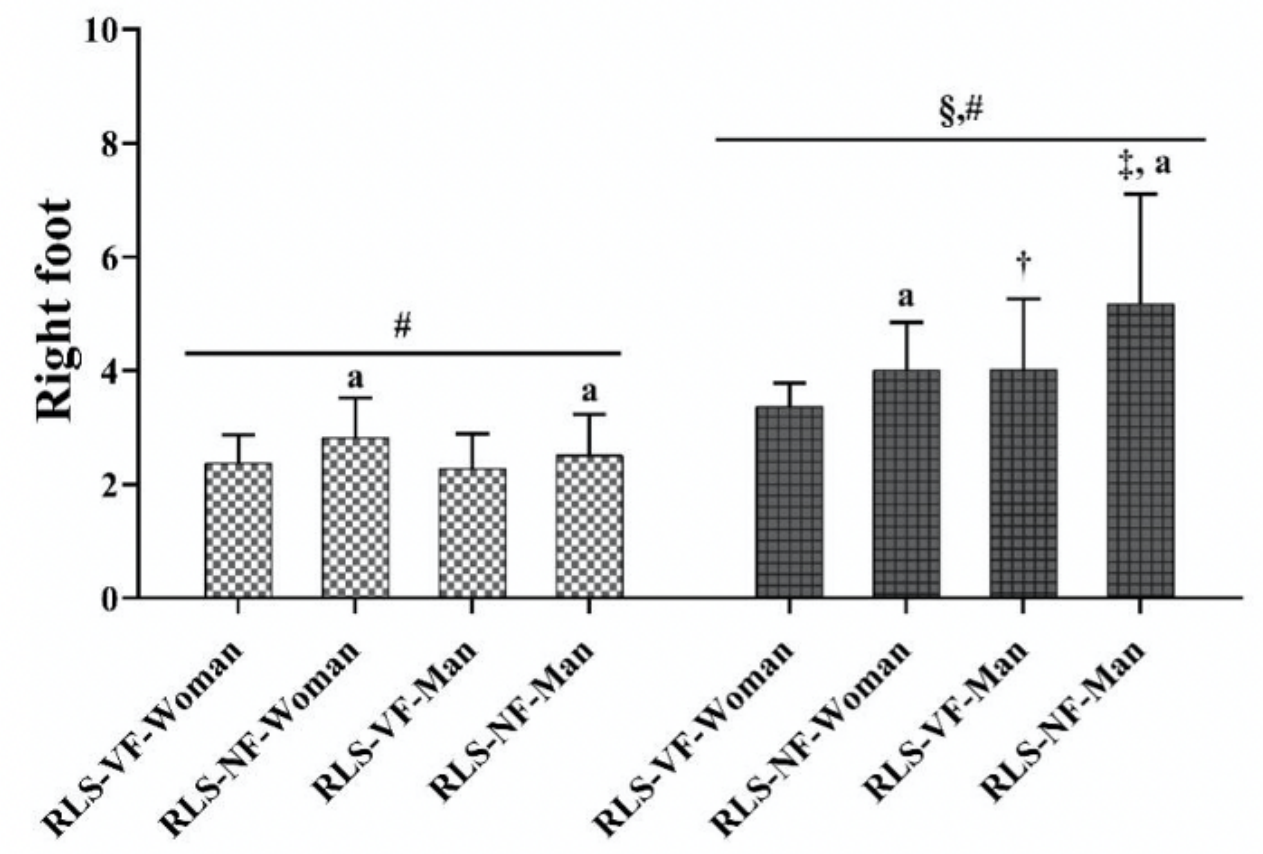

Q COP ML max displacement $(\mathrm{cm})$

$\boxplus$ COP AP max displacement $(\mathrm{cm})$

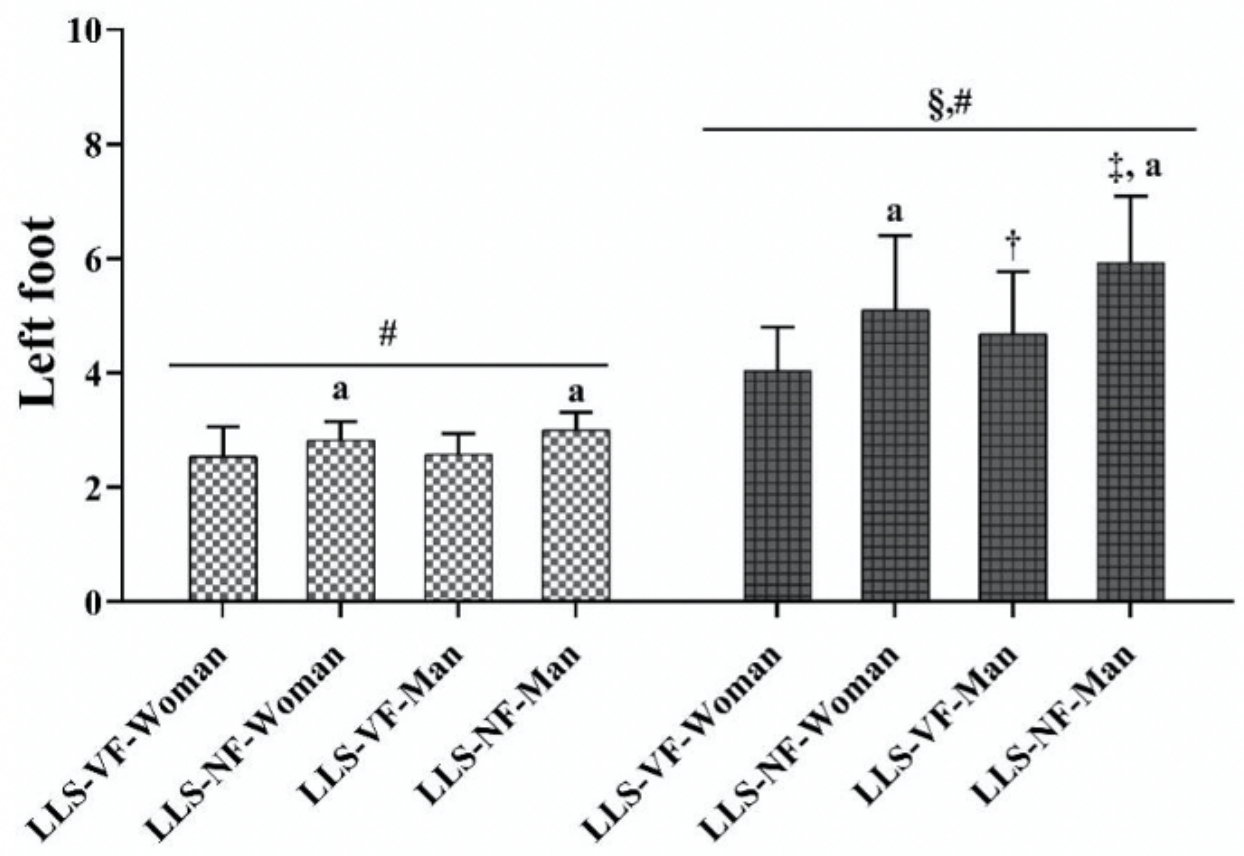

\section{Figure 1}

Gender differences and vision conditions in COPML and COPAL maximum displace-ment parameter * Indicates significant difference in interaction $(p<0.05)$. § Indicates significant differ-ence in main effect (gender) $(p<0.05)$. \# indicates significant difference in main effect (vision conditions) $(p<0.05)$. a indicates significant difference from visual test. $\dagger$ indi-cates significant difference in man and woman's visual test. $¥$ indicates significant dif-ference in men and women's non-visual test. Interaction (vision conditions $\times$ gender) of the COPML maximum displacement or COPAP maximum displacement in RLS/LLS did not differ significantly $(p>0.05)$. (man, $N=20$; woman, $N=20)$ 


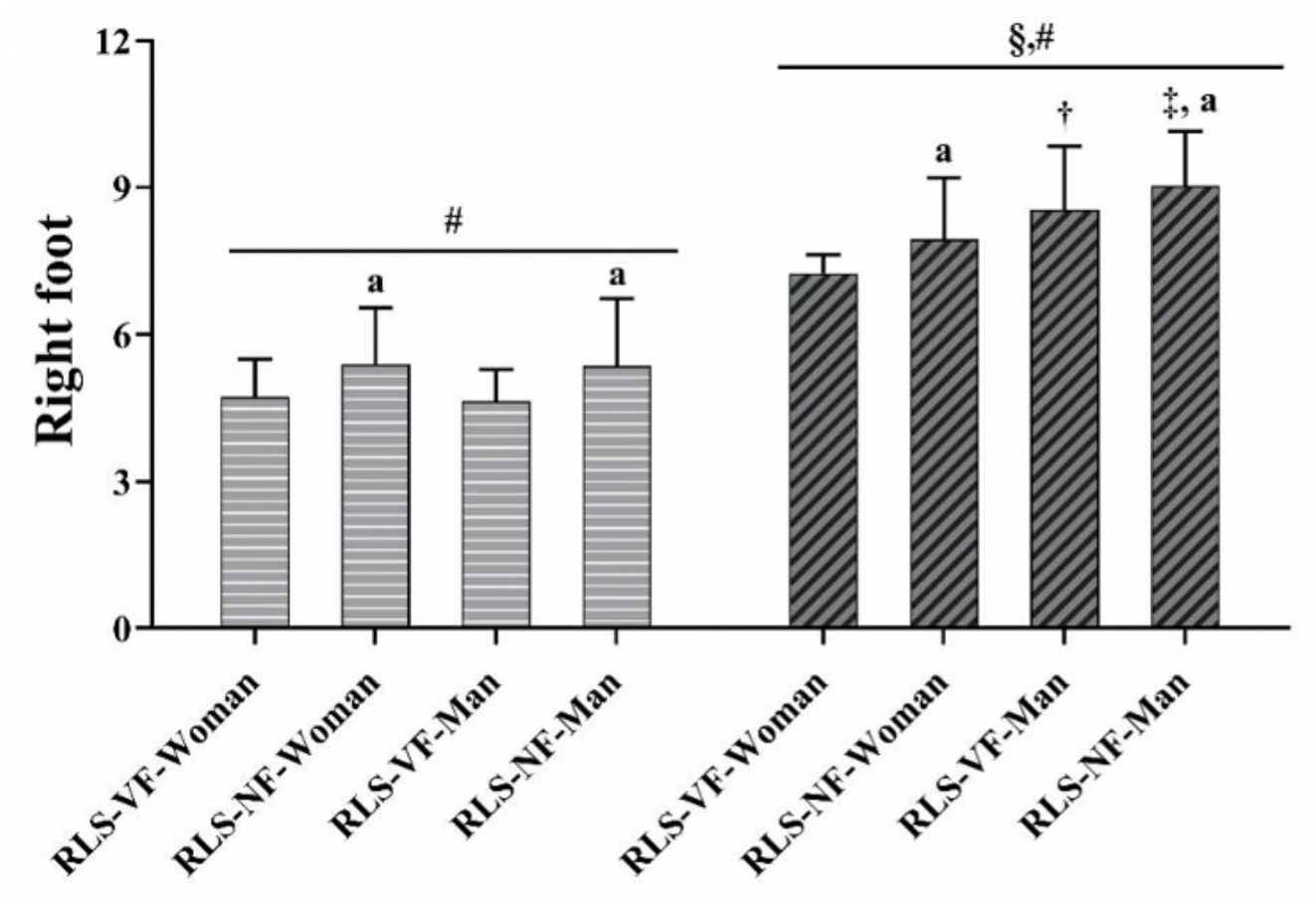

COP ML velocity $(\mathrm{cm} / \mathrm{s})$

COP AP velocity $(\mathrm{cm} / \mathrm{s})$

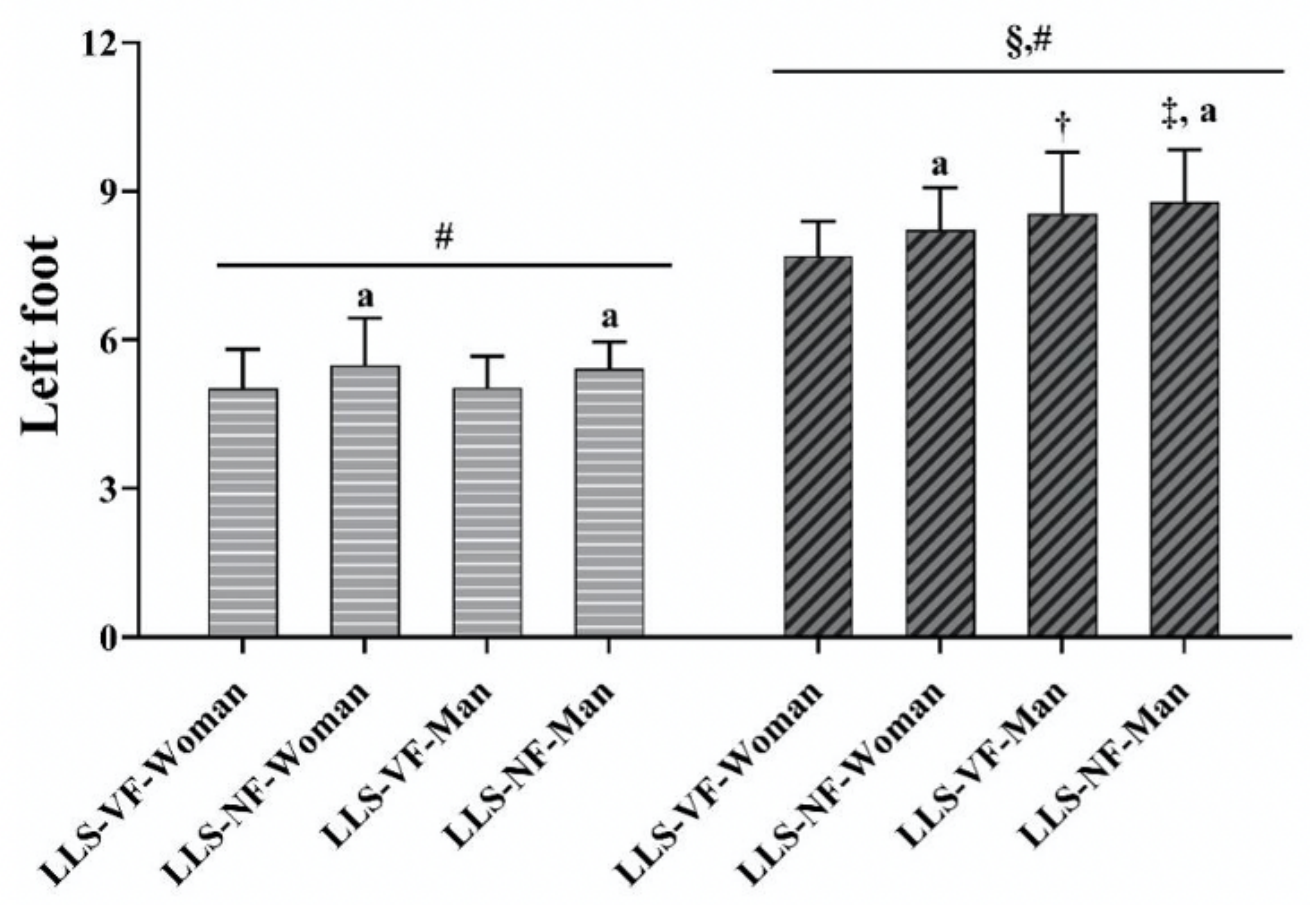

Figure 2

Gender differences and vision conditions in COPML and COPAL velocity parameter * Indicates significant difference in interaction $(p<0.05)$. $§$ Indicates significant differ-ence in main effect (gender) $(p<0.05)$. \# indicates significant difference in main effect (vision conditions) $(p<0.05)$. a indicates significant difference from visual test. $\dagger$ indi-cates significant difference in man and woman's visual test. ‡ indicates significant dif-ference in men and women's non-visual test. Interaction (vision conditions $\times$ gender) of the 
COPML maximum displacement or COPAP maximum displacement in RLS/LLS did not differ significantly $(p>0.05)$. (man, $N=20$; woman, $N=20)$
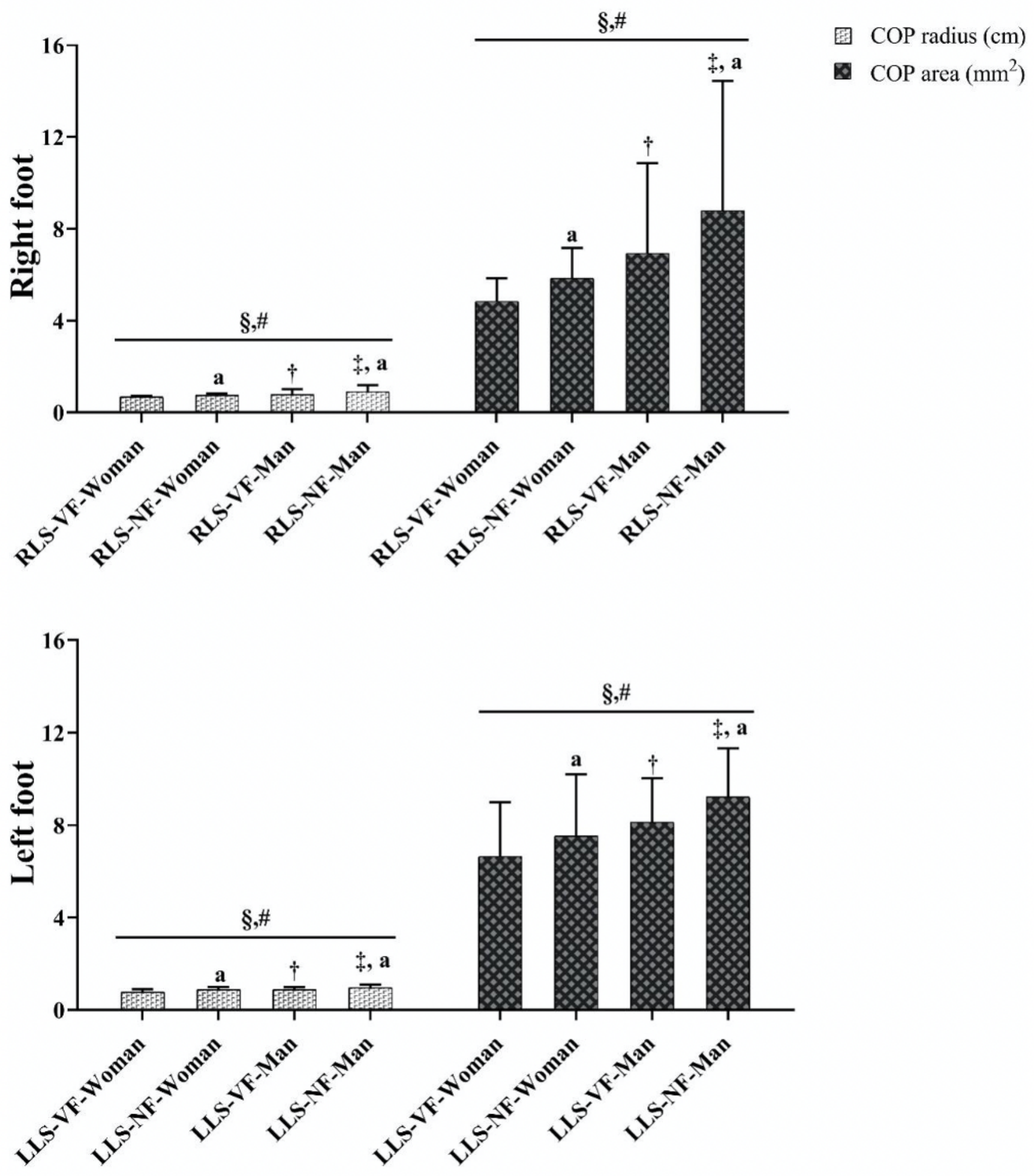

Figure 3

Gender differences and vision conditions in COP radius and COP area parameter * Indicates significant difference in interaction $(p<0.05)$. $\S$ Indicates significant differ-ence in main effect (gender) $(p<0.05)$. \# indicates significant difference in main effect (vision conditions) $(p<0.05)$. a indicates significant 
difference from visual test. $\uparrow$ indi-cates significant difference in man and woman's visual test. $\ddagger$ indicates significant dif-ference in men and women's non-visual test. Interaction (vision conditions $\times$ gender) of the COPML maximum displacement or COPAP maximum displacement in RLS/LLS did not differ significantly $(p>0.05)$. (man, $N=20$; woman, $N=20)$ 